\title{
Utilizing Anesthesiologists, Emergency and Critical Care Physicians with Telemedicine Monitoring to Develop Intubation and Ventilation Services in an Intensive Care Unit in the Austere Medical Environment: A Case Series. Expansion of the EP/CC GAS Project
} \author{
Marc Edson Augustin', Nathalie Edema6, Enzo Del Brocco6, Richard Frechette6, \\ Mark Thompson' ${ }^{1}$, James Corcoran6, Michael Mazowiecki7, Mark Walsh'3,8* \\ ${ }^{1}$ Memorial Hospital Trauma Center, South Bend, IN, USA \\ ${ }^{2}$ University of Maryland School of Medicine, Baltimore, MD, USA \\ ${ }^{3}$ Indiana University School of Medicine, South Bend Campus, South Bend, IN, USA \\ ${ }^{4}$ Mayo Clinic, Rochester, MN, USA \\ ${ }^{5}$ Mayo Clinic, Phoenix, AZ, USA \\ ${ }^{6}$ Hôspital Saint Luc, Port-Au-Prince, Haiti \\ ${ }^{7}$ Excela Health Westmoreland Hospital, Greensburg, PA, USA \\ ${ }^{8}$ Saint Joseph Regional Medical Center, Mishawaka, IN, USA \\ Email: *jdynako@iu.edu, ${ }^{\star}$ markwalshmd@gmail.com
}

Richard Skupski', Arthur Toth', Michael T. McCurdy' ${ }^{2}$, Shane Kappler ${ }^{2}$, James Lantry², Gerson Pyram², Donald Zimmer ${ }^{1}$, Joseph Dynako ${ }^{3 *}$, Anne Grisoli3 ${ }^{3}$, David Zimmer ${ }^{3}$, John Wilson ${ }^{4}$, Bhavesh M. Patel5, Hannelisa Callisen, Alyssa Chapital5, Lovely Nathalie Colas ${ }^{6}$,

How to cite this paper: Skupski, R., Toth, A., McCurdy, M.T., Kappler, S., Lantry, J., Pyram, G., Zimmer, D., Dynako, J., Grisoli, A., Zimmer, D., Wilson, J., Patel, B.M., Callisen, H., Chapital, A., Colas, L.N., Augustin, M.E., Edema, N., Del Brocco, E., Frechette, R., Thompson, M., Corcoran, J., Mazowiecki, M. and Walsh, M. (2018) Utilizing Anesthesiologists, Emergency and Critical Care Physicians with Telemedicine Monitoring to Develop Intubation and Ventilation Services in an Intensive Care Unit in the Austere Medical Environment: A Case Series. Expansion of the EP/CC GAS Project. Open Journal of Anesthesiology, 8, 183-197.

https://doi.org/10.4236/ojanes.2018.86019

\section{Abstract}

Background: Significant resource constraints and critical care training gaps are responsible for the limited development of intensive care units (ICUs) in resource limited settings. We describe the implementation of an ICU in Haiti and report the successes and difficulties encountered throughout the process. We present a consecutive case series investigating an anesthesiologist, emergency, and critical care physician implemented endotracheal intubation and mechanical ventilation protocol in an austere environment with the assistance of telemedicine. Methods: A consecutive case series of fifteen patients admitted to an ICU at St. Luc Hospital located in Port-au-Prince, Haiti, between the months of February 2012 to April 2014 is reported. Causes of respiratory failure and the clinical course are presented. Patients were followed to either death or discharge. Results: Fifteen patients (eight women and seven men) were in- 
Received: May 29, 2018

Accepted: June 24, 2018

Published: June 29, 2018

Copyright (c) 2018 by authors and Scientific Research Publishing Inc. This work is licensed under the Creative Commons Attribution International License (CC BY 4.0).

http://creativecommons.org/licenses/by/4.0/

\section{Open Access}

cluded in the study with an average age of 37.7 years. The mean duration of ventilation was three days. Of the fifteen patients intubated, five patients (33.3\%) survived and were discharged from the ICU. Of the five surviving patients, two were intubated for status epilepticus, one for status asthmaticus and one for hyperosmolar coma associated with intracerebral hemorrhage. Of the patients dying on the ventilator, four patients died from pneumonia, two from renal failure, and one from tetanus. The remaining three died from strokes and cardiac arrests. Conclusions: Mortality of mechanically ventilated patients in a resource-limited country is significant. Focused training in core critical care skills aimed at increasing the endotracheal intubation and ventilatory management capacity of local medical staff should be a priority in order to continue to develop ICUs in these austere environments. Collaborative educational and training efforts directed by anesthesiologists, emergency, and critical care physicians, and aided by telemedicine can facilitate realizing this goal.

\section{Keywords}

Intensive Care Unit (ICU), Critical Care, Mechanical Ventilation, Endotracheal Ventilation, Anesthesiologist, Low and Moderate-Income Country, Austere Environment, Telemedicine

\section{Introduction}

The intensive care unit (ICU) is demanding in skill and breadth of interventions. Few ICUs exist in resource-limited countries because of the cost and extensive spectrum of medical interventions required. We present the implementation of an ICU in Haiti, guided by anesthesiologists, emergency, and critical care physicians, with the assistance of telemedicine, for the provision of an endotracheal intubation and ventilation protocol, and report the successes and difficulties encountered through a description of a pilot program.

\subsection{Background on St. Luc Hospital and History of the Intensive Care Unit}

\subsubsection{History of St. Luc Hospital}

St. Luc Hospital is a 100-bed facility. In 2011, it served over 60,000 patients, including 20,000 cholera patients. The hospital employs over 300 Haitian staff and it offers services in outpatient care as well as a 24-hour emergency department, infectious diseases wards, inpatient wards, and a critical care unit. Long-term housing and care is available for those patients with chronic conditions such as neurological injuries and tuberculosis. A surgery center provides elective general surgical services and intermittent orthopaedic surgical care from visiting US and local Haitian surgeons. Short-term medical personnel from the Mayo Clinic, the University of Maryland Medical Center, and Memorial Hospital of South Bend Indiana, provided clinical training and educational development with local staff. Telemedicine supported these endeavors and allowed for ongoing training and 
feedback.

\subsubsection{History of ICU}

In January of 2012, St. Luc's ICU opened with eight beds, the result of a collaborative effort between the St. Luc Hospital staff and the Mayo Clinic. The three main challenges that needed to be overcome were: 1) equipping and organizing the unit, 2) training doctors and nurses with no prior experience in ICU level care, and 3) reinforcing this training while providing a framework of adapted policies and procedures to guide operations. Donated equipment was set-up and organized with assistance from the Mayo staff. This equipment included cardiac monitors, portable electronic X-ray, two portable LTV1200 ventilators, a bedside ultrasound, IV pumps, CPAP machines, portable suction, electrocardiogram, I-STAT rapid chemistry analyzers, a crash cart, airway equipment materials, and supplies for IV access and medication administration. A system was developed whereby ICU technicians would record supplies that were used and help facilitate restocking supplies in the ICU.

Mayo nurses trained St. Luc's nurses regarding intravenous (IV) access and maintenance, bedside suction, the regular recording of vital signs, the close monitoring of patients, and a protocol for responding to alarms. Physicians were trained in the use of bedside ultrasound, endotracheal intubation, and the administration of mechanical ventilation with the portable LTV1200 ventilators. Technicians were taught how to operate portable X-Ray and EKG machines and perform rapid chemistry analysis. A Mayo pharmacist provided a list of essential ICU medicines, many of which the staff had no prior experience with and provided instructions on how to administer them. The ICU staff and St. Luc Hospital triage nurses received training regarding the recognition of patients needing ICU level care. The ICU has implemented the use of the quick Sequential (Sepsis-Related) Organ Failure Assessment (qSOFA) guidelines to identify early sepsis in patients in austere environments [1]. Simulations of common clinical scenarios, including sepsis, respiratory distress, and acute cardiac arrest, were performed.

Special protocols and policies were developed to guide and reinforce the ICU training. The Mayo Clinic provided ICU admission criteria, which was translated and adapted by St. Luc's medical staff for their unique setting. A basic electronic medical record-keeping system using word processing, with templates for admission and progress notes was implemented. Policies were created on discharges, transfers, and hand-offs. The hospital developed a rounding system whereby the day shift ICU physician would round each morning with the previous night Emergency Department (ED) and ICU physicians on every ED patient in order to determine which patient needed ICU-level care. Before this protocol, there was no ability to provide endotracheal intubation or mechanical ventilation. Anesthesiologists from Memorial Hospital of South Bend were recruited to provide initial training to the Haitian critical care physicians as part of this pilot project. 


\subsubsection{Development of Mechanical Ventilation Program}

Contemporaneous with the evolution of the ICU was the development and implementation of airway management and mechanical ventilation. Intubation and ventilator management represented the most challenging clinical problem for a new ICU staff with limited practical experience in this area. This was further complicated by the austere setting, without typical monitoring aids such as arterial blood gas measurements and basic laboratory availability. Two anesthesiologists, two emergency physicians, and five critical care physicians periodically visited Haiti two to three times during the year for one-week "staggered" blocks to instruct the core group of physicians who staff the emergency department and critical care unit. They focused on specific instruction of endotracheal intubation using direct laryngoscopy (DL) with and without a bougie. Initial instruction was performed on manikins and cadavers and on critically ill patients under the direction of anesthesiologists, emergency physicians, and critical care physicians for the early cases. For the early cases, Haitian physicians who treated patients with endotracheal intubated and mechanical ventilation consulted by telemedicine, FaceTime, and e-mail with the University of Maryland Department of Critical Care and Memorial Hospital in order to assist in the guidance of management. Recent literature has demonstrated the utility of telemedicine to guide ultrasonography by non-physicians in the austere environment and to direct and monitor therapy [2] [3]. Similar approaches were taken with this endeavor.

Fifteen patients were intubated and ventilated during this period, eight women and seven men (Table 1). The average age was 37.7 years old. The mean duration of ventilation was three days. Most intubated patients were sedated except for the very sick neurological cases who did not require sedation. The most commonly reported complication of mechanical ventilation was the occurrence of bed sores which occurred in four patients. One ventilator-associated pneumonia (VAP) was reported, which occurred in the patient with the longest ventilation duration (twelve days). Five patients were successfully extubated and discharged from the ICU alive. These patients were all intubated for respiratory and neurological reasons.

The 5 survivors' diagnoses and ages were: CVA with diabetic hyperosmolar coma, age 56; 2 status epilepticus, (with CVA) age 54 and (with eclampsia) age 27; status asthmaticus, age 45; and tetanus, age 51 . The underlying diseases, rather than the ages, determined survival since the average age of those patients who died was 29.2 years old and the average age of those patients who survived was 46.6 years old. The deaths for the younger group was a function of the severity of the underlying illnesses, such as HIV, sickle cell anemia, chronic alcoholism with cocaine use, cardiac arrest in a severe diabetic, severe diabetic ketoacidosis, massive hypertensive intracerebral hemorrhage, and brain stem hemorrhage due to eclampsia. Of equal importance in the consideration for endotracheal intubation was the likelihood of recovery. Patients who survived had an average length of intubation of 3.0 days, whereas the length of intubation in the patients who failed to survive was 3.28 days. Therefore, there was an attempt 
Table 1. All patients who were on the ventilator in the ICU.

\begin{tabular}{|c|c|c|c|c|c|c|c|c|c|}
\hline $\begin{array}{l}\text { Number of } \\
\text { patient }\end{array}$ & Sex & Age & $\begin{array}{l}\text { Medical } \\
\text { history }\end{array}$ & $\begin{array}{c}\text { Reason for } \\
\text { admission in ICU }\end{array}$ & $\begin{array}{c}\text { Days of } \\
\text { ventilation }\end{array}$ & Sedation & ARF & $\begin{array}{l}\text { Ventilation } \\
\text { complication }\end{array}$ & Death \\
\hline 1 & $\mathrm{~F}$ & 22 & $\begin{array}{l}\text { Sickle Cell } \\
\text { Anemia } \\
\text { tetanus }\end{array}$ & $\begin{array}{l}\text { Respiratory and neu- } \\
\text { rologic failures }\end{array}$ & 12 & Yes & Yes & VAP Day 4 & Dead at D12 \\
\hline 2 & M & 56 & $\begin{array}{l}\text { HTN, CVA, } \\
\text { Diabetes }\end{array}$ & $\begin{array}{l}\text { Neurologic failure } \\
\text { from CVA and } \\
\text { hyperosmolar coma }\end{array}$ & 3 & Yes & Yes & $\begin{array}{l}\text { Repeated } \\
\text { atelectasis }\end{array}$ & $\begin{array}{c}\text { Discharged at } \\
\text { D15 }\end{array}$ \\
\hline 3 & M & $\begin{array}{c}\text { Not } \\
\text { Available }\end{array}$ & $\begin{array}{l}\text { Alcoholism, } \\
\text { COPD }\end{array}$ & $\begin{array}{l}\text { Pleuropneumonia } \\
\text { with respiratory fail- } \\
\text { ure }\end{array}$ & 5 & Yes & No & No & Dead at D6 \\
\hline 4 & $\mathrm{~F}$ & 45 & HTN, HIV & $\begin{array}{c}\text { Massive } \\
\text { hemorrhagic CVA } \\
\text { with neurologic fail- } \\
\text { ure }\end{array}$ & 2 & No & No & No & Dead at D6 \\
\hline 5 & M & 32 & HIV & $\begin{array}{l}\text { Suspected } \\
\text { pneumocystis with } \\
\text { respiratory failure }\end{array}$ & 3 & Yes & No & No & Dead at D3 \\
\hline 6 & $\mathrm{~F}$ & 36 & HIV & $\begin{array}{l}\text { Suspected } \\
\text { pneumocystis with } \\
\text { respiratory failure }\end{array}$ & 3 & Yes & No & Yes & Dead at D1 \\
\hline 7 & M & 42 & $\begin{array}{l}\text { Alcoholism, } \\
\text { cocaine addict }\end{array}$ & $\begin{array}{l}\text { Pneumonia with } \\
\text { respiratory failure }\end{array}$ & 2 & Yes & No & No & Dead at D7 \\
\hline 8 & $\mathrm{~F}$ & 54 & HTN, CVA & $\begin{array}{l}\text { SE with neurologic } \\
\text { failure }\end{array}$ & 3 & Yes & No & Bed sore & $\begin{array}{c}\text { Discharged at } \\
\text { D3 }\end{array}$ \\
\hline 9 & M & 19 & Diabetic & $\begin{array}{l}\text { Ketoacidosis with } \\
\text { neurologic failure }\end{array}$ & $1 / 2$ & Yes & No & Latex allergy & Dead at D1 \\
\hline 10 & F & 42 & CVA, eclampsia & $\begin{array}{l}\text { Hemorrhagic CVA of } \\
\text { brainstem with neu- } \\
\text { rologic failure }\end{array}$ & $1 / 2$ & No & No & No & Dead at D1 \\
\hline 11 & $\mathrm{~F}$ & 27 & None & $\begin{array}{c}\text { Eclampsia, } \\
\text { hypernatremia, CVA } \\
\text { with } \mathrm{SE}\end{array}$ & 10 & Yes & No & No & $\begin{array}{c}\text { Discharged at } \\
\text { D12 }\end{array}$ \\
\hline 12 & $\mathrm{~F}$ & 18 & None & $\begin{array}{l}\text { Intoxication with } \\
\text { neurologic failure }\end{array}$ & 2 & No & Yes & No & Dead at D4 \\
\hline 13 & M & 45 & Asthma & Status Asthmaticus & 4 & Yes & No & Bed sore & Discharged D9 \\
\hline 14 & $\mathrm{~F}$ & 52 & Diabetic & Cardiac arrest & 2 & No & No & No & Dead at D2 \\
\hline 15 & M & 51 & Tetanus & $\begin{array}{l}\text { Respiratory and neu- } \\
\text { rologic failure }\end{array}$ & 4 & Yes & Yes & No & $\begin{array}{l}\text { Discharge } \\
\text { D4 }\end{array}$ \\
\hline
\end{tabular}

CVA: Cerebrovascular Accident. HTN: Hypertension. ARF: Acute Renal Failure. COPD: Chronic Obstructive Pulmonary Disease. HIV: Human Immunodeficiency Virus. SE: Status Epilepticus. N/A: Not Applicable. In Table 1, we describe patients who received ventilation in the intensive care unit from February 2012 to April 2013. We did not include patients intubated due to cardiac arrest who died within a few hours after intubation.

to not prolong intubation and the decisions to intubate were based not on age, but on the severity of the underlying disease and the chances of return to a normal life. Haiti is a country where hypertension and diabetes have a strong genet- 
ic preponderance and represent a high majority of patients who are part of this case series. Notice that four of the fifteen patients did not receive any sedation and these patients predictably died. The average length of sedation for these patients was 3.25 days.

\section{Objective and Methods}

The objective of this paper is to describe the development of an ICU in the austere environment under the guidance of anesthesiologists, emergency and critical care physicians, with particular emphasis on the instruction of endotracheal intubation and ventilator management for critically ill patients in need of acute bridge ventilation until recovery.

A consecutive case series of fifteen patients admitted to an ICU at St. Luc Hospital located in Port-au-Prince, Haiti, between the months of February 2012 to April 2014 who required mechanical ventilation is presented. Causes of respiratory failure and the clinical course were recorded. Patients were followed to either death or discharge. Patients intubated less than four hours were excluded from the study.

\section{Case Series}

Of the fifteen patients intubated and ventilated by Haitian critical care physicians, five survived. These five patients were in excellent health prior to their illnesses and had a high likelihood of survival after a brief period of intubation and ventilation. We provide examples of two cases below who are emblematic of the type of patients that we expect will benefit from this type of intervention in an austere environment, where this type of patient with a readily curable illness might otherwise die.

\subsection{Sample Case: Patient 11}

In February 2013, a 27-year-old woman presented to the emergency department at St. Luc for repeated seizures and agitation lasting for 4 hours. She was 13 days post-partum. Upon arrival to the emergency department, her Glasgow score was 15 with no obvious sensorimotor deficits. Vital signs follow: blood pressure $170 / 115 \mathrm{mmHg}$, pulse 110 , respiratory rate 22 , temperature $38^{\circ} \mathrm{C}$, and her blood sugar was normal. Shortly after admission she had seizures again controlled by 5 mg of diazepam IV. A CT scanner, donated by the Mayo Clinic-was utilized to obtain a CT scan of the head and the patient was found to have an intracerebral bleed (Figure 1).

The patient progressed to status epilepticus. She was transferred to the ICU, where she was sedated, intubated, and ventilated by Haitian critical care physicians to reduce her seizures and protect her airway. She received multiple boluses of propofol and a midazolam drip. Her seizures resolved immediately.

She was mechanically ventilated with a LTV1200. The settings of the LTV1200 were: tidal volume $600 \mathrm{ml}$, respiratory rate 12, fraction of inspired oxygen starting 


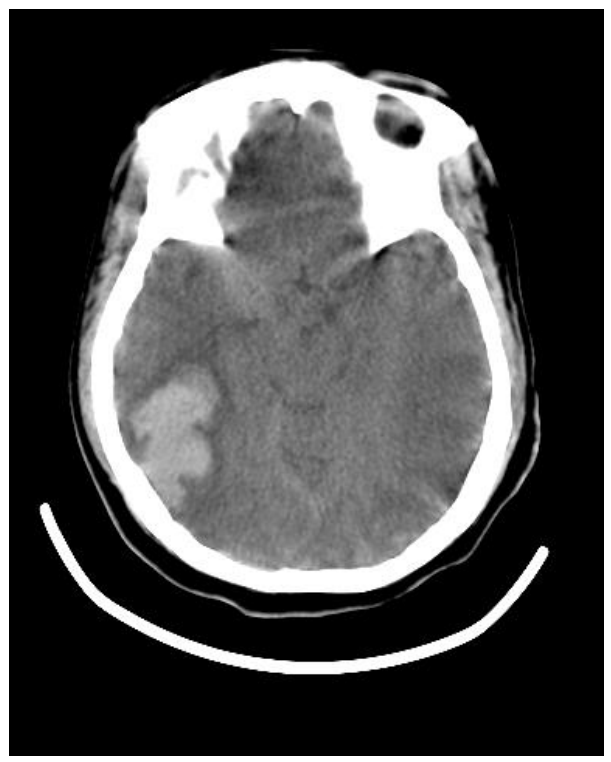

Figure 1. CT of the brain of a patient number 11 , a 27 year old with eclampsia, intracerebral hemorrhage and hypernatremia who survived and walked out of the ICU after 12 days mechanical ventilation.

at $100 \%$ and titrated based on oxygen saturation, positive end expiratory pressure $5 \mathrm{mmHg}$.

The electrolytes at admission into the ICU found hypernatremia of 179 $\mathrm{mmol} / \mathrm{L}$ (due to a three-day delay for laboratory tests results). This was corrected by intravenous hypotonic solution and free water in the nasogastric tube. She was also treated with ceftriaxone and metronidazole for suspected aspiration pneumonia, and chloroquine for malaria (given the findings of fever and seizures in a malaria endemic country).

The patient's clinical evolution was favorable under sedation. She experienced her last seizure 3 hours after intubation. After 24 hours of sedation, she was seizure-free. The hypernatremia was corrected. At that time, the decision was made to end sedation. She awakened quickly after cessation of midazolam and responded to simple commands. The extubation was successful. The neurological examination post-extubation did not find any sensorimotor deficit but noted an aphasia which resolved within 24 hours. The patient was discharged 72 hours after ICU admission with a Glasgow Coma Scale score of 15 and was given phenytoin for future seizure prophylaxis and made full recovery.

\subsection{Sample Case: Patient 15}

A 51-year-old man presented to the ED at St. Luc with a complaint of increasing muscle weakness. The patient had a facial grimace, drooling, stridor, and general muscular rigidity, which are classic signs for tetanus. The patient was intubated and ventilated by local Haitian critical care physicians after sedation with midazolam and paralysis with rocuronium. The patient received intravenous antibio- 
tics and anti-tetanus toxoid as well as continued sedation and paralysis with lorazepam and vecuronium. With SMS text messaging, e-mail, and Facetime connection to the University of Maryland Department of Critical Care Medicine, the patient was managed for four days on an LTV1200 portable mechanical ventilator without the benefit of an arterial blood gas (ABG). On the fifth day, with the arrival of an anesthesiologist and two emergency physicians who had participated in the initial education of the physicians and staff in the ICU of St. Luc Hospital, the patient was extubated by the Haitian staff. He was subsequently discharged on the sixth day in normal health (Figure 2). This entire case was managed by local Haitian physicians who had been trained by United States anesthesiologists, emergency, and critical care physicians. This successful management of tetanus solely by Haitian critical care physicians, who provided endotracheal intubation and ventilation, was possible because of the experience gained from the first index case of a much more severely ill tetanus patient. Index patient number 1 was a 22 -year-old patient with severe tetanus who subsequently died after endotracheal intubation by a Haitian critical care physician and ventilation for ten days, which was managed by Haitian critical care physicians, with periodic consultation by telemedicine. This index patient number 1 served as a template upon which successful care was provided for the less severely affected tetanus patient number 15 (Figure 3).

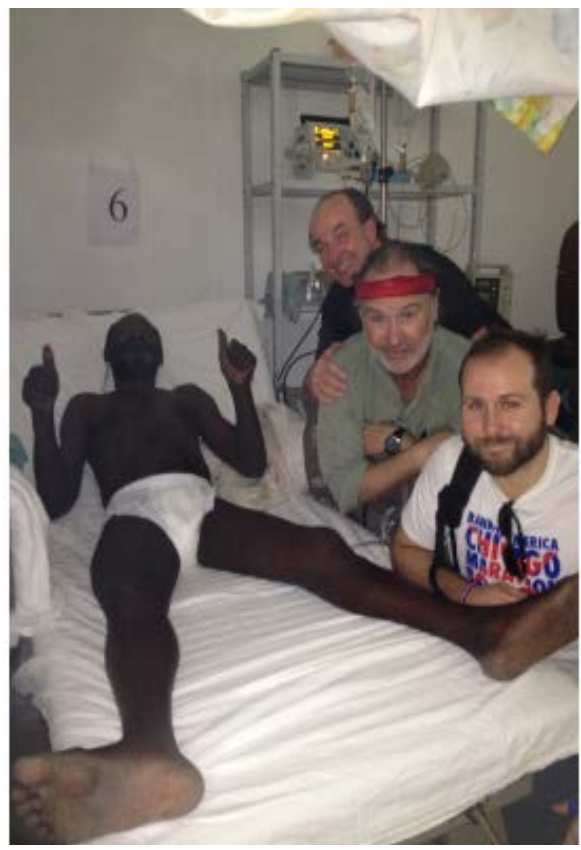

Figure 2. Patient number 15, a 51 yer old man who was successfully intubated and ventialted in the ICU by Haitian intnesivists for tetanus. 4 days of ventilation management was asssited with telemedicine. This picture was taken after extubation and after arrival of initial superiving anesthesiologist and emergency phsyciains. 


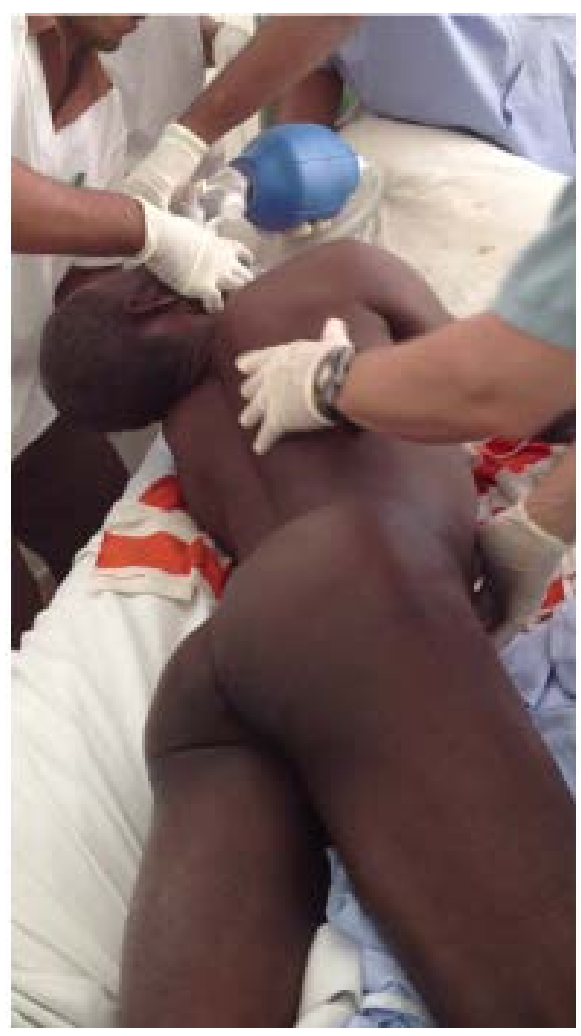

Figure 3. Patient number 1, a 22-year-old man who later succumbed to tetanus. Bag mask ventilation prior to endotracheal intubation by the Haitian intensivist with the guidance of the supervising anesthesiologist.

\section{Analysis}

\subsection{Application of ICU Technology in the Austere Environment: Obstacles to Overcome}

There were predictable and significant problems encountered during the implementation period involving endotracheal intubation and ventilation in this recently developed ICU, in a country where few ICUs existed before.

Monitoring ICU patients was complicated by the fact that not all equipment monitors were the same. In addition, routine maintenance was problematic and remains a challenge for the future.

The LTV1200 is an excellent tool for bridge ventilation and transport. We have adopted the LTV1200 because of its portability, as well as reliability. Once the ICU staff became familiar with the ventilators, management of these patients became more uniform. We feel, from this pilot case series, that it is essential to have identical ventilators to decrease human error due to multiple ventilator systems. Other authors describe the use of the Siemens 1900 in the austere environment which has the advantage of being an intensive care ventilator, but it is a non-transportable ventilator. For short term ventilations, such as these 15 patients, the LTV1200 was adequate and can be more easily transported to the 
austere environment.

At St. Luc, oxygen is distributed in 2200 PSI tanks. The hospital has its own compressor, with sufficient oxygen available during the daytime hours only. Technicians and doctors have to prepare a reserve supply when there is a ventilated patient for the nighttime hours.

Sedation and paralytic medication administration is complicated by the lack of both central venous access and electronic IV pumps. The staff has resorted to temporary use of intraosseous lines when intravenous access was lost. During the course of the study, the staff has improved in their ability to gain intravenous access with regular continuing education programs that have allowed the staff to improve their ability to obtain peripheral, central venous, and intraosseous access.

Obtaining laboratory data was intermittent. The laboratory was open only during regular business hours during the week, and the laboratory did not run ABG samples. The ISTAT-8's were clinically useful, particularly in patients with metabolic disorders, such as diabetes, hypernatremia, hypokalemia, and metabolic acidosis. This testing allowed the clinicians to search for and treat reversible causes in comatose patients.

Important questions were raised during the implementation of this ICU. There was concern about the utilization of scarce resources to provide ventilation for patients with a marginal prognosis. There was also frequent discussion about the implementation of ventilation without the exhaustion of scarce resources. This question concerning the use of technology and the limitation of admission to the ICU in the austere environment has been previously addressed in the literature [4] [5]. As we have demonstrated, endotracheal intubation and mechanical ventilation with a portable ventilator does not require as complex technology as what might be expected [4]. Furthermore, ICUs in developing countries cannot follow the example of ICUs in developed countries. The patient populations are very different in developing countries and, as we have seen in our case series, the patients are often young with acute illnesses that have a well-described treatment with excellent prognoses. This "inverted demographic composition" of the low-income country patient population has been contrasted with the high utilization of critical care resources in the industrialized world [4]. This situation contrasts with patients in developed countries who typically are elderly with multiple comorbidities and often little likelihood of survival. Yet, considerable resources are expended on these patients who face certain death or guarded prognosis [4]. A focused austere utilization of "feasible care guidelines and protocols (which) can be scaled across low-resource settings" has been proposed in order to effectively treat the younger patient with the better prognosis for a reversible disease in the austere environment [4].

\subsection{Formation}

During the development of the ICU, the training anesthesiologists, emergency, 
and critical care physicians had to confront the fact that the Haitian physicians did not have any prior practical training in critical care medicine. Their only exposure had been theoretical training provided by foreign volunteers, primarily physicians who were only able to speak and teach in English. Overall, the Haitian physicians had some understanding of critical care medicine, but the nurses and technicians had very little understanding of how an ICU operates.

There were significant initial problems in training and skill retention. While the physicians were able to retain intubating skills, there were issues with being able to reprogram the ventilator settings. Adequate response to alarms needed to be emphasized repeatedly. There were concerns about the effective sedation of ventilated patients. One important obstacle was that families in Haiti traditionally take care of the patients' waste (i.e. feces and urine), but in a sedated patient this must be done by the nurse to decrease the risk of extubation. Further, although the hospital possessed an electrical pump for sedative medications, the nursing staff were often unware of its use despite initial instructions. Therefore, patients were given sedation by drip with the intermittent extubation of the patient and the requirement for reintubation which was initially assisted by telemedicine.

Overall, however, through ongoing training of caregivers and staff it was possible to successfully perform mechanical ventilation in this unit with the available equipment and as such significantly improved the prognosis of some patients.

\subsection{Ethics}

There were several ethical considerations for each patient that was intubated. Consideration was always given to the of limited resources (i.e. the ventilator and medication for sedation). For example, the supply of essential materials is not constant. Therefore, the decision had to be made if the patient has a chance to respond to therapy and if there would be adequate supplies and medicines to maintain the patient for a brief period of mechanical ventilation.

Use of the ventilator is an aggressive therapy especially under the conditions in Haiti. A concept that must be taken into consideration is one of prolonged suffering for the patient. There must be a reasonable chance of success when deciding to impose such treatment on the patient.

Finally, the issue of resuscitating patients who survive but become handicapped is important, because most of these patients are coming from families who cannot care for a disabled person. Haiti has little support for economically challenged families or disabled individuals; thus, a discussion with the family must take place prior to intubating these patients in whom there poses a risk of severe disability post-extubation.

\section{Ventilation Implementation}

\subsection{Patient Selection}

It must be clearly established which patients will benefit from ventilation. Venti- 
lation conditions are different in Haiti, as described above, and thus the risk-reward balance of ventilation is very different compared to developed countries.

Based on our surviving patients who had status epilepticus, tetanus, hyperosmolar coma, and eclampsia, for example, have good outcomes and are therefore representative examples of good indications for intubation. Of the patients who died, four of the patients had irreversible pathology such as massive stroke or acute renal insufficiency. Acute renal insufficiency is an irreversible disease in Haiti because dialysis machines are not widely available.

Other patients presented with pneumonia with undetermined pathology, due to the lack of diagnostic means. The two suspected pneumocystis pneumonias in HIV patients died.

\subsection{Early Extubation}

There are multiple complications related to mechanical ventilation. The question remains for how far and how long intubation and mechanical ventilation can be implemented without becoming systematically deleterious under these conditions; namely, lack of training, lack of basic equipment, and difficult monitoring conditions. Therefore, early extubation is important.

\section{Future}

Our case series demonstrates that it is possible to set up an ICU in low resource area, such as in Haiti, and offer mechanical ventilation to critically ill patients.

This technology can be a benefit to patients in critical situations in developing countries who are often younger compared with developed countries [4] [6]. In our case series we found this trend to be true with an average age of 37.7 years old. Since age is an independent risk factor for mortality in mechanical ventilation [7], we think that this young population will benefit from the implemented airway management program.

Cost is a challenge under these conditions. Performing cost-effectiveness studies in order to have a real idea of the price of mechanical ventilation in such conditions would be beneficial for future care decisions. Although there is data from developed countries, it is impossible to extrapolate this data because the monitoring equipment is very different. For example, a study estimated that for patients on mechanical ventilation in an ICU in Nepal it costs an additional $\$ 40$ per day [8], compared with $\$ 10,794$ in another study conducted in the USA [9]. This discrepancy indicates that ventilator technology might not be as expensive as it seems.

Additionally, ICU-level care could improve the public perception of the hospital by improving the survival of critical patients. It has been shown that improving the hospital image improves the population's confidence and makes them more receptive to public health measures [10] [11].

Our experience shows that technical difficulties can be overcome, and that it is 
not necessary to seek the same conditions as those of the developed countries in order to mechanically ventilate a patient. Observational studies which include larger patient cohorts will better identify the real beneficiaries from mechanical ventilation. This will help in determining indications for intubation in the ICU of developing countries.

The limitations of this consecutive case series are that this is an isolated application of a program that depends on a very intense and frequent intervention by the anesthesiologist, emergency, and critical care physician in the initial education and then continued maintenance of endotracheal intubation and ventilation management skills of the local Haitian physicians. The continued maintenance of skills requires long distance learning via FaceTime, e-mail, SMS text messaging, and telemedicine. This program is clearly not standardized and will be unique depending on the needs and frequency of intervention by the anesthesiologist, emergency, and critical care physician. However, the program has provided a general template for the successful implementation and continued operation of a successful ICU in the austere environment.

The training of the staff remains most problematic issue [12]. Concentrated efforts and resources must be well directed in order to guarantee competent ventilation in the ICU. Regarding this particular concern, the anesthesiologists have joined forces with the critical care physicians and emergency physicians in the United States, with the critical care physicians and anesthesiologists in Haiti, to build a surgery program whereby US physicians teach Haitian physicans the skills of endotracheal intubation during surgical cases in the operating rooms which are attached to the ICU. This unique resource has resulted in more than 1700 cases of surgery where the anesthesia was initially performed by emergency physicians and critical care physicians under the guidance of anesthesiologists from the US. Within the last few years St. Luc has been able to recruit anesthesiologists who now perform most of the anesthesia in those two operating rooms. This close proximity of the anesthesiologist to the critical care physicians allows for continued educational opportunity to maintain endotracheal intubation skills.

As a result of the above-mentioned collaboration between local Haitian surgeons, anesthesiologists, and critical care physicians, and US anesthesiologists, emergency physicians and critical care physicians, a curriculum has been developed which allows the anesthesiologist, emergency, and critical care physician to instruct the Haitian critical care physicians in all aspects of airway management. Since 2013, this methodology and its curriculum has been based on published case series of emergency physicians, guided by anesthesiologists, performing general anesthesia in the austere environments in Haiti. This curriculum which has now been published in anesthesia as well as orthopaedic journals is known as the Emergency Physicians General Anesthesia Syllabus (EP GAS) [13]. This EP GAS curriculum has been applied to more than 170 orthopaedic cases and has been published as the Orthopaedic General Anesthesia Syllabus (ORTHO-GAS) and has expanded to critical care physicians with the Critical 
Care General Anesthetic Syllabus (CC-GAS) [14]. Future publications will include the instructions by anesthesiologists of critical care physicians from the Mayo Clinic and the University of Maryland, who have similarly provided anesthesia in the austere environment as well as taught critical care physicians in Haiti how to perform endotracheal intubation and manage patients on ventilators. Finally, a recent grant from the Mayo Clinic in Rochester Minnesota, with participation of United States based physicians, nurses and physician's pssistants has allowed the critical care physicians in Haiti to provide an annual conference in Haiti, titled: "The Haitian Acute Care and Emergency Care Conference," which is organized by the critical care physicians at St. Luc for Haitian physicians (http://haecc.org/2018-program/).

\section{Conclusion}

We report our experiences of endotracheal intubation and mechanical ventilation in a country with limited resources. Some challenges with techniques and staff training were encountered but overcome with additional focus on ongoing training. This critical care advance has permitted the survival of patients who formerly died, especially with short-term ventilation indications. Future studies should include cost-benefit analyses, as well as further observational studies. Used together, this data would improve patient selection so that resources can be conserved while saving the maximal number of lives. Strengthening staff training in the ICU should be prioritized in order to continue to develop mechanical ventilation and the ICU in Haiti. It is not uncommon for beginning programs which provide and establish new medical advances in the austere environment of low income countries to fail. We describe an anesthesiologist, emergency, and critical care physician directed program which provides initial, practical, and continued educational opportunities for endotracheal intubation and ventilator management on rudimentary and temporary ventilators. This program has survived since its inception in 2013 and may offer a template for the utilization of anesthesiologist, emergency, and critical care physician to not only initiate complete airway management in the critical care setting, but also to provide a method for continued success and development in the austere environment.

\section{Acknowledgements}

The family of Stefan Randjelovic for their support of this educational program and Ross McCauley, Vincente Shah, Nuha Zackariya and Faadil Sharrif for editorial assistance.

\section{References}

[1] Rudd, K.E., Seymour, C.W., Aluisio, A.R., et al. (2018) Association of the Quick Sequential (Sepsis-Related) Organ Failure Assessment (qSOFA) Score With Excess Hospital Mortality in Adults With Suspected Infection in Low-and Middle-Income Countries. JAMA. 
[2] Levine, A.R., Buchner, J.A., Verceles, A.C., et al. (2016) Ultrasound Images Transmitted via FaceTime Are Non-Inferior to Images on the Ultrasound Machine. Journal of Critical Care, 33, 51-55. https://doi.org/10.1016/j.jcrc.2016.02.019

[3] Levine, A.R., McCurdy, M.T., Zubrow, M.T., Papali, A., Mallemat, H.A. and Verceles, A.C. (2015) Tele-Intensivists Can Instruct Non-Physicians to Acquire High-Quality Ultrasound Images. Journal of Critical Care, 30, 871-875. https://doi.org/10.1016/j.jcrc.2015.05.030

[4] Adhikari, N.K., Fowler, R.A., Bhagwanjee, S. and Rubenfeld, G.D. (2010) Critical Care and the Global Burden of Critical Illness in Adults. The Lancet, 376, 1339-1346. https://doi.org/10.1016/S0140-6736(10)60446-1

[5] Watters, D.A.K., Wilson, I.H., Leaver, R.J. and Bagshawe, A., Eds. (1991) Care of the Critically Ill Patient in the Tropics and Subtropics. Macmillan Education, Basingstoke, $450 \mathrm{p}$.

[6] Jochberger, S., Ismailova, F., Lederer, W., et al. (2008) Anesthesia and Its Allied Disciplines in the Developing World: A Nationwide Survey of the Republic of Zambia. Anesthesia \& Analgesia, 106, 942-948.

https://doi.org/10.1213/ane.0b013e318166ecb8

[7] Azevedo, L.C., Park, M., Salluh, J.I., et al. (2013) Clinical Outcomes of Patients Requiring Ventilatory Support in Brazilian Intensive Care Units: A Multicenter, Prospective, Cohort Study. Critical Care, 17, R63. https://doi.org/10.1186/cc12594

[8] Basnet, S., Adhikari, N. and Koirala, J. (2011) Challenges in Setting up Pediatric and Neonatal Intensive Care Units in a Resource-Limited Country. Pediatrics, 128, e986-e992. https://doi.org/10.1542/peds.2010-3657

[9] Dasta, J.F., McLaughlin, T.P., Mody, S.H. and Piech, C.T. (2005) Daily Cost of an Intensive Care Unit Day: The Contribution of Mechanical Ventilation. Critical Care Medicine, 33, 1266-1271. https://doi.org/10.1097/01.CCM.0000164543.14619.00

[10] Razzak, J.A. and Kellermann, A.L. (2002) Emergency Medical Care in Developing Countries: Is It Worthwhile? Bulletin of the World Health Organization, 80, 900-905.

[11] Organization, W.H. (2000) The World Health Report 2000: Health Systems: Improving Performance. World Health Organization.

[12] Riviello, E.D., Letchford, S., Achieng, L. and Newton, M.W. (2011) Critical Care in Resource-Poor Settings: Lessons Learned and Future Directions. Critical Care Medicine, 39, 860-867. https://doi.org/10.1097/CCM.0b013e318206d6d5

[13] Skupski, R., Walsh, M., Jbara, M., et al. (2014) The Training and Performance of Emergency Physicians as Anesthetists for International Medical Surgical Response Teams: The Emergency Physician's General Anesthesia Syllabus (EP GAS). Open Journal of Anesthesiology, 4, 53. https://doi.org/10.4236/ojanes.2014.42009

[14] Pierre, O., Lovejoy Jr., J.F., Stanton, R., et al. (2018) The Use of Emergency Physicians to Deliver Anesthesia for Orthopaedic Surgery in Austere Environments: The Expansion of the Emergency Physician's General Anesthesia Syllabus to Orthopaedic Surgery. JBJS, 100, e44. https://doi.org/10.2106/JBJS.16.01481 